\title{
Generalized $\star$-products, Wilson lines and the solution of the Seiberg-Witten equations
}

\section{Thomas Mehen}

Physics Department, Ohio State University, Columbus OH 43210, USA

E-mail: 'mehen@mps.ohio-state.edu'

\section{Mark B. Wise}

California Institute of Technology, Pasadena, CA 91125, USA

E-mail: iwisètheory.caitech.èdui

ABSTRACT: Higher order terms in the effective action of non-commutative gauge theories exhibit generalizations of the $\star$-product (e.g. $\star^{\prime}$ and $\star_{3}$ ). These terms do not manifestly respect the non-commutative gauge invariance of the tree level action. In $\mathrm{U}(1)$ gauge theories, we note that these generalized $\star$-products occur in the expansion of some quantities that are invariant under non-commutative gauge transformations, but contain an infinite number of powers of the non-commutative gauge field. One example is an open Wilson line. Another is the expression for a commutative field strength tensor $F_{a b}$ in terms of the non-commutative gauge field $\widehat{A}_{a}$. Seiberg and Witten derived differential equations that relate commutative and non-commutative gauge transformations, gauge fields and field strengths. In the $\mathrm{U}(1)$ case we solve these equations neglecting terms of fourth order in $\widehat{A}$ but keeping all orders in the non-commutative parameter $\theta^{k l}$.

KEywords: Nonperturbātive Effects, Non-Commutative Geometryi. 
Non-commutative field theories are quantum field theories which live on a spacetime in which the coordinates do not commute:

$$
\left[x^{i}, x^{j}\right]=i \theta^{i j} .
$$

Non-commutative gauge theories emerge naturally when considering the low energy limit of open strings in the presence of a background $B$-field [i] - The low energy effective action is obtained by considering the tree level scattering of massless open string states. The zero slope limit is taken in such a way that the open string met-

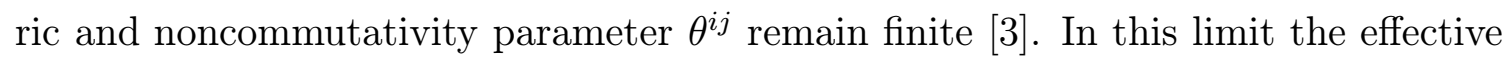
action for the non-commutative gauge theory can be obtained from that of the ordinary gauge theory simply by replacing ordinary products of fields with Moyal or $\star$-products. The $\star$-product is defined by:

$$
f(x) \star g(x)=\left.\exp \left[\frac{i}{2} \theta^{i j} \frac{\partial}{\partial x^{i}} \frac{\partial}{\partial y^{j}}\right] f(x) g(y)\right|_{x=y} .
$$

For instance, the non-commutative $\mathrm{U}(1)$ gauge theory action is

$$
S=-\frac{1}{4 g^{2}} \int d^{4} x \widehat{F}_{a b} \star \widehat{F}^{a b}
$$

where the non-commutative gauge field strength is

$$
\widehat{F}_{a b}=\partial_{a} \widehat{A}_{b}-\partial_{b} \widehat{A}_{a}-i\left[\widehat{A}_{a}, \star \widehat{A}_{b}\right] .
$$

We have introduced the notation $[A, \star B] \equiv A \star B-B \star A$. In eq. (离), $\left[A_{a}, \star A_{b}\right]$ does not vanish because the $\star$-product is non commutative. The action in eq. (יㅣㅁ) is invariant under the infinitesmal non-commutative gauge transformation

$$
\begin{aligned}
\delta_{\widehat{\lambda}} \widehat{A}_{a} & =\partial_{a} \widehat{\lambda}+i\left[\widehat{\lambda}, \star \widehat{A}_{a}\right] \\
\delta_{\widehat{\lambda}} \widehat{F}_{a b} & =i\left[\widehat{\lambda}, \star \widehat{F}_{a b}\right] .
\end{aligned}
$$

Non-commutative field theories exhibit peculiar phenomena unlike that of local

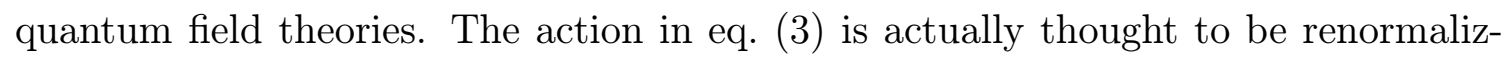
able [8]- [20] even though it contains an infinite number of higher derivative operators. Another peculiar phenomenon is UV-IR mixing [2] 1$]-[25]$. The commutation relation in eq. (i $(i-1)$ gives rise to an uncertainty relation which forces objects which are localized over a short distance in one space direction to be spread out over a long distance in an orthogonal direction. Thus UV and IR modes of the theory are linked and the usual decoupling of the ultraviolet from the infrared does not occur in these theories.

The appearance of Moyal products in the action of non-commutative gauge the-

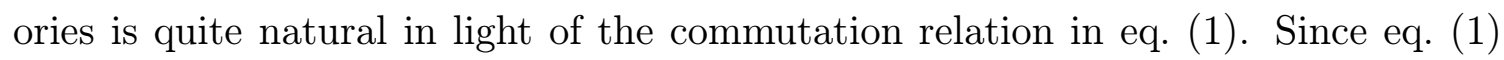
is essentially identical to the commutation relation of annihilation and creation operators, there is an isomorphism between functions $f$ of ordinary coordinates and 
operators $\mathcal{O}_{f}$ in the Hilbert space of annihilation and creation operators. To define the map from ordinary functions to operators in Hilbert space, a prescription for specifying the ordering of annihilation and creation operators in $\mathcal{O}_{f}$ is needed. If Weyl ordering is used to define this map, then it is easy to show that the Moyal product of functions is isomorphic to ordinary operator multiplication (see e.g. [26iㅣ).

Higher order terms in the effective action for massless open string fields 2 2:에, certain global anomalies in U(1) non-commutative gauge theories coupled to matter [29] and the coupling of open strings to closed strings in the presence of a background $B$-field [30 than what is seen at tree level. Instead of the Moyal products the higher order contributions to the effective action contain generalized $\star$-products such as

$$
f(x) \star^{\prime} g(x)=\left.\frac{\sin \left(\frac{\partial_{1} \wedge \partial_{2}}{2}\right)}{\frac{\partial_{1} \wedge \partial_{2}}{2}} f\left(x_{1}\right) g\left(x_{2}\right)\right|_{x_{i}=x}
$$

and

$$
\begin{aligned}
{[f(x) g(x) h(x)] \star_{3}=} & {\left[\frac{\sin \left(\frac{\partial_{2} \wedge \partial_{3}}{2}\right) \sin \left(\frac{\partial_{1} \wedge\left(\partial_{2}+\partial_{3}\right)}{2}\right)}{\frac{\left(\partial_{1}+\partial_{2}\right) \wedge \partial_{3}}{2} \frac{\partial_{1} \wedge\left(\partial_{2}+\partial_{3}\right)}{2}}+(1 \leftrightarrow 2)\right] \times } \\
& \times\left. f\left(x_{1}\right) g\left(x_{2}\right) h\left(x_{3}\right)\right|_{x_{i}=x},
\end{aligned}
$$

where

$$
\partial_{1} \wedge \partial_{2}=\theta^{i j} \frac{\partial}{\partial x_{1}^{i}} \frac{\partial}{\partial x_{2}^{j}} .
$$

Note that $\star^{\prime}$ is symmetric in $f$ and $g$ and $\star_{3}$ is invariant under all permutations of $f, g$ and $h$, though this may not be obvious from eq. (i $\left.i_{1}^{1} i\right)$.

The appearance of these generalized $\star$-products in the effective action is somewhat confusing. First of all, the effective action does not manifestly respect the non-commutative $\mathrm{U}(1)$ gauge symmetry of eq. ( $\left(\underline{b}^{\prime}\right)$. It is hard to believe that radiative corrections do not respect the gauge symmetry of the tree level action. Second, while the Moyal product can be simply understood as a consequence of the commutation relation in eq. (ii i $)$, the nature of these generalized $\star$-products remains unclear.

In this paper, we point out that the generalized $\star$-products also appear in the expansion of gauge invariant operators constructed in $\left[\begin{array}{l}{[3} \\ 2\end{array}\right.$ local operators attached to open Wilson lines. Expanding the Wilson line to $O\left(\widehat{A}^{2}\right)$ in

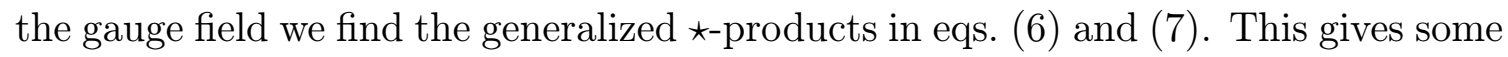

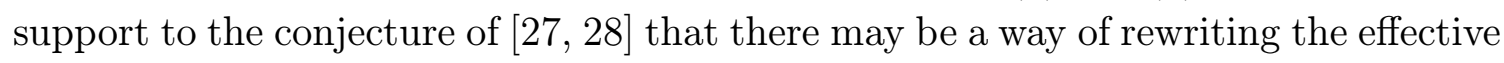
action so that the non-commutative gauge symmetry is manifest. We also point out that a specific gauge invariant operator is closely related to the solutions of the Seiberg-Witten (SW) differential equation for the U(1) gauge theory. The solution 
of SW equation is a map between ordinary gauge fields and non-commutative gauge fields which preserves the gauge equivalence classes of the respective theories. We present a solution of the SW equation which is correct to all orders in $\theta^{i j}$ and to $O\left(\widehat{A}^{3}\right)$ in the gauge field. This solution also exhibits the generalized $\star$-products.

We begin with a brief review of the gauge invariant operators introduced in 13. (see also [3. adjoint representation has no local gauge invariant operators. There are non-local gauge invariant operators which are the Fourier transform of local operators attached to an open Wilson line.

The Wilson line is defined by

$$
W(x, C)=P_{\star} \exp \left(-i \int_{0}^{1} d \sigma \frac{d \zeta^{i}}{d \sigma} \widehat{A}_{i}(x+\zeta(\sigma))\right),
$$

where $C$ denotes path parametrized by $\zeta^{i}(\sigma)$, such that $\zeta^{i}(0)=0$ and $\zeta^{i}(1)=l^{i}$. $P_{\star}$ denotes the usual path ordering with ordinary products of fields replaced by $\star$-products. The Wilson line transforms under non-commutative U(1) gauge transformations as

$$
W(x, C) \longrightarrow \mathrm{U}(x) \star W(x, C) \star U(x+l)^{\dagger} .
$$

Now consider a local operator constructed from the non-commutative gauge field which transforms as

$$
\widehat{\mathcal{O}} \longrightarrow \mathrm{U}(x) \star \widehat{\mathcal{O}} \star U(x)^{\dagger} .
$$

An example of such an operator is $\widehat{\mathcal{O}}=\hat{F}_{a b}$. The operator $\widetilde{\mathcal{O}}$ with Fourier transform

$$
\widetilde{\mathcal{O}}^{(\mathrm{FT})}=\int d^{4} x \widehat{\mathcal{O}} \star W(x, C) \star e^{i k x}
$$

is invariant under non-commutative gauge transformations [i $[\overline{3} 2 \overline{2}, \overline{3} \overline{3} 3]$ provided we choose $l^{i}=\theta^{i j} k_{j}$.

Expanding the gauge invariant operator $\widetilde{\mathcal{O}}$ to $O\left(\widehat{A}^{2}\right)$, we obtain

$$
\begin{aligned}
\widetilde{\mathcal{O}}^{(\mathrm{FT})}=\int d^{4} x \widehat{\mathcal{O}} \star & W(x, C) \star e^{i k x} \\
=\int d^{4} x \widehat{\mathcal{O}} \star & {\left[1-i \int_{0}^{1} d \sigma \frac{d \zeta^{i}}{d \sigma} \widehat{A}_{i}(x+\zeta(\sigma))-\right.} \\
& \left.\quad-\int_{0}^{1} d \sigma_{1} \int_{\sigma_{1}}^{1} d \sigma_{2} \frac{d \zeta^{j}}{d \sigma_{1}} \frac{d \zeta^{k}}{d \sigma_{2}} \widehat{A}_{j}\left(x+\zeta\left(\sigma_{1}\right)\right) \star \widehat{A}_{i}\left(x+\zeta\left(\sigma_{2}\right)\right)+\cdots\right] \star e^{i k x} .
\end{aligned}
$$

The result of doing these integrals depends on the path chosen. We will choose the path corresponding to a straight Wilson line: $\zeta^{i}(\sigma)=\theta^{i j} k_{j} \sigma$. We then use

$$
\widehat{A}_{i}(x+\theta k \sigma)=e^{-\sigma k \wedge \partial} \widehat{A}_{i}(x) .
$$


The integrals are over $\sigma_{i}$ are simple and it is not hard to show that

$$
\widetilde{\mathcal{O}}^{(\mathrm{FT})}=\int d^{4} x\left[\widehat{\mathcal{O}}+\theta^{i j} \partial_{j}\left(\widehat{\mathcal{O}} \star^{\prime} \widehat{A}_{i}\right)+\frac{1}{2} \theta^{i j} \theta^{k l} \partial_{j} \partial_{l}\left[\widehat{\mathcal{O}} \widehat{A}_{i} \widehat{A}_{k}\right] \star_{3}+\cdots\right] e^{i k x} .
$$

It is straightforward to check that the series inside the brackets of eq. (1) $1 \overline{5}$ invariant up to terms of $O\left(\widehat{A}^{2}\right)$. Two identities which are useful for this are

$$
\begin{aligned}
\theta^{i j} \partial_{i} f \star^{\prime} \partial_{j} g & =-i[f, \star g], \\
f \star^{\prime}[g, \star h]+g \star^{\prime}[f, \star h] & =i \theta^{i j} \partial_{i}\left[f g \partial_{j} h\right] \star_{3} .
\end{aligned}
$$

Even though the definition of $\widetilde{O}$ was given in terms of ordinary $\star$-products its expansion in powers of the non-commutative gauge field involves $\star^{\prime}$ - and $\star_{3}$-products.

Next we briefly discuss the Seiberg-Witten map between ordinary gauge fields and non-commutative gauge fields and show that the generalized $\star$-products also appear in this map.

Non-commutative Yang-Mills is in fact equivalent to ordinary gauge theory perturbed by an infinite number of higher dimension operators. It is possible to show that there exists a map between non-commutative gauge fields and ordinary gauge fields which preserves the gauge equivalence classes of the respective theories, despite

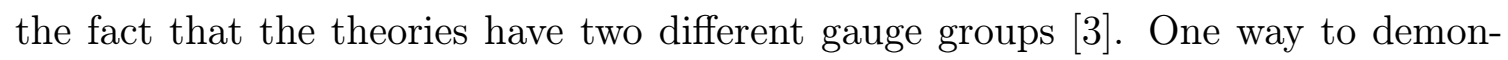
strate this is to show that the two space-time theories can be obtained from the same world sheet sigma model regulated in two different ways.

The relationship between the commutative gauge field and field strength and the analogous non-commutative quantities depends on the parameter $\theta^{k l}$. For $\theta^{k l}=0$, $F_{a b}=\widehat{F}_{a b}$ and $A_{a}=\widehat{A}_{a}$. It is possible derive differential equations for the gauge field, gauge parameter and field strength as functions of $\theta^{k l}$ : [․ㅣㅁ]

$$
\begin{aligned}
\delta \widehat{A}_{a}= & -\frac{1}{4} \delta \theta^{k l}\left[\widehat{A}_{k} \star\left(\partial_{l} \widehat{A}_{a}+\widehat{F}_{l a}\right)+\left(\partial_{l} \widehat{A}_{a}+\widehat{F}_{l a}\right) \star \widehat{A}_{k}\right] \\
\delta \widehat{\lambda}= & \frac{1}{4} \delta \theta^{k l}\left(\partial_{k} \widehat{\lambda} \star \widehat{A}_{l}+\widehat{A}_{l} \star \partial_{k} \widehat{\lambda}\right) \\
\delta \widehat{F}_{a b}=\frac{1}{4} \delta \theta^{k l} & {\left[2 \widehat{F}_{a k} \star \widehat{F}_{b l}+2 \widehat{F}_{b l} \star \widehat{F}_{a k}-\widehat{A}_{k} \star\left(\widehat{D}_{l} \widehat{F}_{a b}+\partial_{l} \widehat{F}_{a b}\right)-\right.} \\
& \left.-\left(\widehat{D}_{l} \widehat{F}_{a b}+\partial_{l} \widehat{F}_{a b}\right) \star \widehat{A}_{k}\right] .
\end{aligned}
$$

In the remainder of this paper, we will refer to these equations as the Seiberg-Witten (SW) equations.

In [3i] the SW equations were solved for the special case of constant $\mathrm{U}(1)$ fields. In $[\overline{3} \overline{0} \overline{0}]$, the equations were solved to $O\left(\widehat{A}^{2}\right)$ by integrating along a special path in the space of matrices $\theta^{k l}$. Specifically, [3] 0 then integrates $\alpha$ from 0 to 1 with the boundary condition $\widehat{F}_{a b}(\alpha=0)=F_{a b}, \widehat{A}_{a}(\alpha=$ $0)=A_{a}$ and $\widehat{\lambda}(\alpha=0)=\lambda$. Note that the solution obtained by integrating the SW 
equation depends on the path of integration [3.5. However, the path dependence can be absorbed entirely into a field redefinition of the non-commutative gauge field. For the case of a $\mathrm{U}(1)$ gauge theory, the solution for the field strength tensor is

$$
F_{a b}=\widehat{F}_{a b}+\theta^{k l}\left(\widehat{A}_{k} \star^{\prime} \partial_{l} \widehat{F}_{a b}-\widehat{F}_{a k} \star^{\prime} \widehat{F}_{b l}\right)+O\left(\widehat{A}^{3}\right) .
$$

The result is exact in $\theta$ and correct to order $O\left(\widehat{A}^{2}\right)$ in the fields. The l.h.s. of eq. (18 is invariant under the ordinary $\mathrm{U}(1)$ gauge transformation. Since the SW map preserves gauge equivalence classes, the r.h.s. of eq. (1) $\left.\bar{\phi}_{1}\right)$ should be invariant under non-commutative $\mathrm{U}(1)$ gauge transformations to $O(\widehat{\hat{A}})$. It is straightforward to check that this is the case.

Integrating the SW equations to $O\left(\widehat{A}^{2}\right)$ is straightforward because at this order one can neglect the $\theta^{k l}$ dependence of $\widehat{A}_{a}$ and $\widehat{F}_{a b}$ in eq. (i1 $\left.\bar{z}_{i}\right)$. Direct integration of the SW equations is difficult at higher orders in $\widehat{A}$. Below we will give a method which allows one to obtain higher order solutions to the SW equations.

This method exploits the similarity of the solution to the SW equation and the gauge invariant operator:

$$
\begin{aligned}
\widetilde{F}_{a b}^{(\mathrm{FT})} & =\int d^{4} x \widehat{F}_{a b} \star W(x, C) \star e^{i k x} \\
& =\int d^{4} x\left[\widehat{F}_{a b}+\theta^{k l} \partial_{l}\left(\widehat{A}_{k} \star^{\prime} \widehat{F}_{a b}\right)+\cdots\right] e^{i k x} \\
& =\int d^{4} x\left[\widehat{F}_{a b}+\theta^{k l}\left(\widehat{A}_{k} \star^{\prime} \partial_{l} \widehat{F}_{a b}+\frac{1}{2} \widehat{F}_{l k} \star^{\prime} \widehat{F}_{a b}\right)+\cdots\right] e^{i k x} .
\end{aligned}
$$

Notice that one of the order $O\left(\widehat{A}^{2}\right)$ terms in the expansion of $\widetilde{F}_{a b}$ is identical to an $O\left(\widehat{A}^{2}\right)$ term in the solution to the SW equation for $F_{a b}$ given in eq. (i $\left.\overline{1}_{-1}\right)$. This is because both $\widetilde{F}_{a b}$ and $F_{a b}$ are gauge invariant, and the term $\theta^{k l} \widehat{A}_{k} \star^{\prime} \partial_{l} \widehat{F}_{a b}$ is necessary to ensure gauge invariance to $O(\widehat{A}) . \quad \widetilde{F}_{a b}$ and $F_{a b}$ differ by terms which are by themselves gauge invariant to $O(\widehat{A})$. A crucial difference between $\widetilde{F}_{a b}$ and $F_{a b}$ is that $F_{a b}$ is a field strength and therefore obeys the Bianchi identity while $\widetilde{F}_{a b}$ does not.

Using what we know about the gauge invariant operator $\widetilde{F}_{a b}$ it is possible to construct the $O\left(\widehat{A}^{3}\right)$ solution to the SW equations. To the $O\left(\widehat{A}^{2}\right)$ solution to the SW equations for the field strength given in eq. (1) we add the $O\left(\widehat{A}^{3}\right)$ term in the expansion of $\widetilde{F}_{a b}$

$$
\begin{aligned}
\widehat{F}_{a b}+ & \theta^{i j} \partial_{j}\left(\widehat{A}_{i} \star^{\prime} \widehat{F}_{a b}\right)+\frac{1}{2} \theta^{i j} \theta^{k l} \partial_{i} \partial_{k}\left[\widehat{F}_{a b} \widehat{A}_{j} \widehat{A}_{l}\right] \star_{3}+ \\
& +\theta^{i j}\left(\frac{1}{2} \widehat{F}_{a b} \star^{\prime} \widehat{F}_{i j}-\widehat{F}_{a i} \star^{\prime} \widehat{F}_{b j}\right) .
\end{aligned}
$$

The first three terms are gauge invariant to $O\left(\widehat{A}^{2}\right)$. The remaining terms under a gauge transformation give rise to an $O\left(\widehat{\lambda} \widehat{F}^{2}\right)$ term, which can be cancelled by adding 


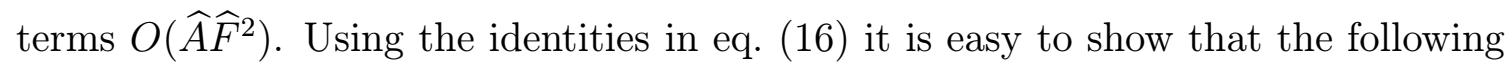
quantity is gauge invariant to $O\left(\widehat{A}^{2}\right)$ :

$$
\begin{aligned}
\widehat{F}_{a b}+ & \theta^{i j}\left(\partial_{j}\left(\widehat{A}_{i} \star^{\prime} \widehat{F}_{a b}\right)+\frac{1}{2} \widehat{F}_{a b} \star^{\prime} \widehat{F}_{i j}-\widehat{F}_{a i} \star^{\prime} \widehat{F}_{b j}\right)+ \\
& +\frac{1}{2} \theta^{i j} \theta^{k l}\left(\partial_{i} \partial_{k}\left[\widehat{F}_{a b} \widehat{A}_{l} \widehat{A}_{j}\right] \star_{3}-\partial_{k}\left[\widehat{F}_{i j} \widehat{F}_{a b} \widehat{A}_{l}\right] \star_{3}+2 \partial_{k}\left[\widehat{F}_{a i} \widehat{F}_{b j} \widehat{A}_{l}\right] \star_{3}\right) .
\end{aligned}
$$

Equation $(\underline{1} \overline{2} \overline{1}-1)$ can be written as

$$
\begin{aligned}
\theta^{i j} \theta^{k l} & \left(\frac{1}{2}\left[\widehat{F}_{a i} \widehat{F}_{b j} \widehat{F}_{k l}\right] \star_{3}-\frac{1}{8}\left[\widehat{F}_{a b} \widehat{F}_{i j} \widehat{F}_{k l}\right] \star_{3}-\frac{1}{4}\left[\widehat{F}_{a b} \widehat{F}_{j k} \widehat{F}_{i l}\right] \star_{3}+\left[\widehat{F}_{i k} \widehat{F}_{a l} \widehat{F}_{b j}\right] \star_{3}\right)+ \\
& +\partial_{a} A_{b}-\partial_{b} A_{a}
\end{aligned}
$$

where

$$
\begin{aligned}
A_{b}= & \widehat{A}_{b}+\frac{1}{2} \theta^{i j} \widehat{A}_{i} \star^{\prime}\left(\partial_{j} \widehat{A}_{b}+\widehat{F}_{j b}\right)+ \\
& +\frac{1}{2} \theta^{i j} \theta^{k l}\left[-\widehat{A}_{i} \partial_{k} \widehat{A}_{b}\left(\partial_{j} \widehat{A}_{l}+\widehat{F}_{j l}\right)+\partial_{k} \partial_{i} \widehat{A}_{b} \widehat{A}_{j} \widehat{A}_{l}+2 \partial_{k} \widehat{A}_{i} \partial_{b} \widehat{A}_{j} \widehat{A}_{l}\right] \star_{3}+O\left(\widehat{A}^{4}\right) .
\end{aligned}
$$

Since eq. ( $(2 \overline{1})$ is gauge invariant to $O\left(\widehat{A}^{2}\right), A_{b}$ must transform like an ordinary $\mathrm{U}(1)$ gauge field up to terms $O\left(\widehat{A}^{3}\right)$. Hence, $A_{b}$ is a solution to the SW differential equation for the gauge field. Explicitly $\delta_{\lambda} A_{b}=\partial_{b} \lambda$ where,

$$
\lambda=\widehat{\lambda}+\frac{1}{2} \theta^{i j} \widehat{A}_{i} \star^{\prime} \partial_{j} \widehat{\lambda}+\frac{1}{2} \theta^{i j} \theta^{k l}\left[\partial_{k} \partial_{i} \widehat{\lambda} \widehat{A}_{j} \widehat{A}_{l}+\partial_{k} \widehat{\lambda} \widehat{A}_{i} \partial_{l} \widehat{A}_{j}\right] \star_{3}+O\left(\widehat{A}^{3}\right) .
$$

$\lambda$ in eq. ( $(2 \overline{4})$ is the solution to the $\mathrm{SW}$ differential equation for the gauge parameter. The field strength constructed from the gauge field in eq. (니히), $F_{a b}=\partial_{a} A_{b}-\partial_{b} A_{a}$, is gauge invariant to $O\left(\widehat{A}^{2}\right)$ and obeys the Bianchi identity. Using our previous results it is easy to show that

$$
\begin{aligned}
F_{a b}= & \widehat{F}_{a b}+\theta^{i j}\left(\partial_{j}\left(\widehat{A}_{i} \star^{\prime} \widehat{F}_{a b}\right)+\frac{1}{2} \widehat{F}_{a b} \star^{\prime} \widehat{F}_{i j}-\widehat{F}_{a i} \star^{\prime} \widehat{F}_{b j}\right)+ \\
& +\frac{1}{2} \theta^{i j} \theta^{k l}\left(\partial_{i} \partial_{k}\left[\widehat{F}_{a b} \widehat{A}_{i} \widehat{A}_{j}\right] \star_{3}-\partial_{k}\left[\widehat{F}_{i j} \widehat{F}_{a b} \widehat{A}_{l}\right] \star_{3}+2 \partial_{k}\left[\widehat{F}_{a i} \widehat{F}_{b j} \widehat{A}_{l}\right] \star_{3}\right)- \\
& -\theta^{i j} \theta^{k l}\left(\frac{1}{2}\left[\widehat{F}_{a i} \widehat{F}_{b j} \widehat{F}_{k l}\right] \star_{3}-\frac{1}{8}\left[\widehat{F}_{a b} \widehat{F}_{i j} \widehat{F}_{k l}\right] \star_{3}-\frac{1}{4}\left[\widehat{F}_{a b} \widehat{F}_{j k} \widehat{F}_{i l}\right] \star_{3}+\left[\widehat{F}_{i k} \widehat{F}_{a l} \widehat{F}_{b j}\right] \star_{3}\right)+ \\
& +O\left(\widehat{A}^{4}\right) .
\end{aligned}
$$

$F_{a b}$ in eq. $\left(2 \underline{2}^{\prime}\right)$ is the solution of the SW differential equation for the field strength.

In [3] commutative gauge fields is derived. Explicit constructions of the Seiberg-Witten

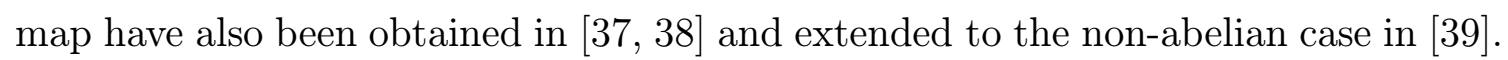
In [3]6], a solution for $\widehat{A}(A)$ to $O\left(A^{3}\right)$ is obtained. The $O\left(A^{2}\right)$ part of the solution in $[\overline{3} \overline{6}]$ is exact in $\theta$ and exhibits the $\star^{\prime}$-product. However, the $O\left(A^{3}\right)$ part of the solution in $[\overline{3} \overline{6} \bar{b}]$ is not exact in $\theta$ so does not have the $\star_{3}$-product. 
The SW equations only involve ordinary $\star$-products. However we have seen that the solution to these equations when expanded in powers of the non-commutative gauge field involve $\star^{\prime}$ - and $\star_{3}$-products. These generalized $\star$-products also appear in the expansion of the gauge invariant Wilson line, as well higher orders in the effective action of non-commutative gauge theories and in the coupling of massless closed string states to non-commutative gauge fields. Though existing calculations [2], 25,28 of higher order terms in the effective action do not respect the non-commutative gauge symmetry of the tree level action, the existence of similar structures in the expansion of gauge invariant non-local quantities suggests that it should be possible to write down gauge invariant, albeit non local, expressions for the effective

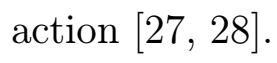

\section{Acknowledgments}

We thank J. Gomis and E. Witten for useful discussions. T.M. was supported by the National Science Foundation under Grant No. PHY-9800964. M.B.W. was supported in part by the Department of Energy under grant DE-FG03-ER-40701.

\section{References}

[1] A. Connes, M.R. Douglas and A. Schwarz, Noncommutative geometry and matrix theory: compactification on tori, "U. High Energy Phys. 02 (1998) 003 [hep-th/9711162].

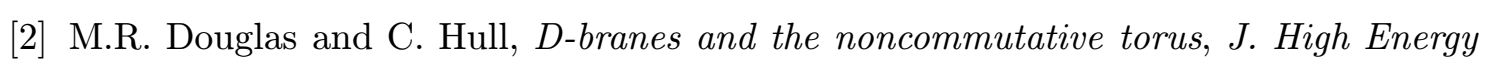

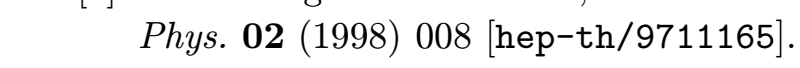

[3] N. Seiberg and E. Witten, String theory and noncommutative geometry, i $\bar{L}$.

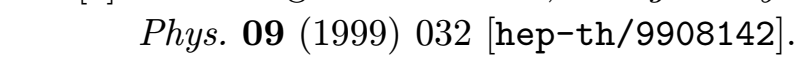

[4] Y.-K.E. Cheung and M. Krogh, Noncommutative geometry from 0-branes in a background B-field, iNucl. Phys. B $\mathbf{5 2 8}$ (1998) $185 ;[$ hêp-th/980303i!].

[5] C.-S. Chu and P.-M. Ho, Noncommutative open string and D-brane, 'iNučcl. $\bar{P} \bar{h} y \bar{s} .1$ :

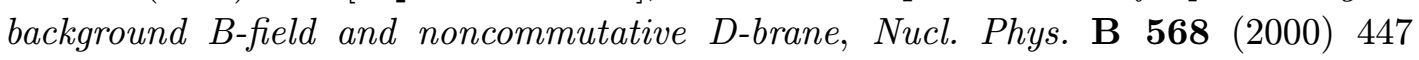
hep-th/9906192in.

[6] V. Schomerus, D-branes and deformation quantization, 'J. :

[7] F. Ardalan, H. Arfaei and M.M. Sheikh-Jabbari, Dirac quantization of open strings and noncommutativity in branes, Nucl. Phys. B $\mathbf{5} 7 \mathbf{6}-(2000)-578$ Mixed branes and $m$ (atrix) theory on noncommutative torus, hep-th/9803067.

[8] T. Filk, Divergencies in a field theory on quantum space, Phys. Lett. (- - - 53 
[9] J.C. Varilly and J.M. Gracia-Bondia, On the ultraviolet behaviour of quantum

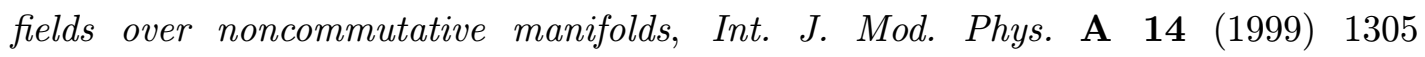
[hep-th/9804001].

[10] M. Chaichian, A. Demichev and P. Presnajder, Quantum field theory on noncommutative space-times and the persistence of ultraviolet divergences, 'Nㅡㄷㅡ.

- 2000 360; [hep-th/9812180]; Quantum field theory on the noncommutative plane with $E_{q}(2)$ symmetry,

[11] C.P. Martin and D. Sanchez-Ruiz, The one-loop uv divergent structure of U(1) YangMills theory on noncommutative $R^{4},{ }^{\prime} \bar{P} h y s$. Rev. Lett. $\mathbf{8 3}(1999) 476$ ' [hep-th/9903077i].

[12] M.M. Sheikh-Jabbari, Renormalizability of the supersymmetric Yang-Mills theories

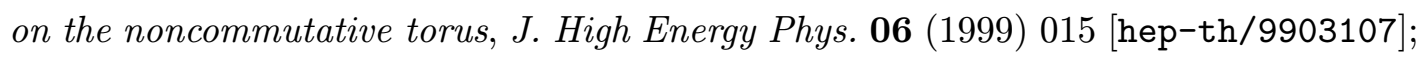
Noncommutative super Yang-Mills theories with 8 supercharges and brane configurations, Nucl. Phys. B 587 (2000) 195: [nep-th/0001089"].

[13] I.Y. Aref'eva, D.M. Belov and A.S. Koshelev, A note on UV/IR for noncommutative complex scalar field, hep-th/0001215'

I.Y. Aref'eva, D.M. Belov, A.S. Koshelev and O.A. Rytchkov, UV/IR mixing for noncommutative complex scalar field theory, 2. Interaction with gauge fields, hep-th/0003176!

[14] T. Krajewski and R. Wulkenhaar, Perturbative quantum gauge fields on the noncommutative torus, 'Int. J. Mod. Phys. A 15 (2000) 1011' [hep-th/9903187i];

H. Grosse, T. Krajewski and R. Wulkenhaar, Renormalization of noncommutative Yang-Mills theories: a simple example, hep-th/0001182.

[15] S. Cho, R. Hinterding, J. Madore and H. Steinacker, Finite field theory on noncommutative geometries, Int. J. Mod. Phys. D9 (2000) 161 [hep-th/9903239'].

[16] E. Hawkins, Noncommutative regularization for the practical man, hep-th/9908052..

[17] I. Chepelev and R. Roiban, Convergence theorem for non-commutative feynman graphs and renormalization, hep-th/0008090.

[18] H.O. Girotti, M. Gomes, V.O. Rivelles and A.J. da Silva, A consistent noncommutative field theory: the Wess-Zumino model, iNucl. Phys. [hep-th/000527̄in.

[19] S.S. Gubser and S.L. Sondhi, Phase structure of non-commutative scalar field theories, hep-th/0006119.

[20] A. Armoni, Comments on perturbative dynamics of non-commutative Yang-Mills theory, iNucl. Phys. B 593 - $(2001) 229$ [hep-th/0005208].

[21] S. Minwalla, M.V. Raamsdonk and N. Seiberg, Noncommutative perturbative dynamics, hep-th/9912072.

[22] S.V. Raamsdonk and N. Seiberg, Comments on noncommutative perturbative dynam-

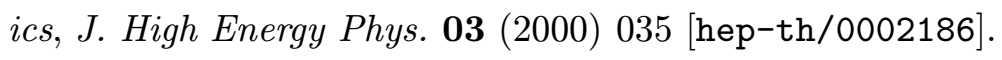


[23] M. Hayakawa, Perturbative analysis on infrared and ultraviolet aspects of noncommutative $Q E D$ on $R^{4}$, hep-th/9912167.

[24] W. Fischler, E. Gorbatov, A. Kashani-Poor, S. Paban, P, Pouliot and J. Gomis, Evidence for winding states in noncommutative quantum field theory, $\overline{\mathrm{J}}$ High Energy :

[25] A. Matusis, L. Susskind and N. Toumbas, The IR/UV connection in the noncommutative gauge theories, nep-th/0002075.

[26] R. Gopakumar, S. Minwalla and A. Strominger, Noncommutative solitons, '

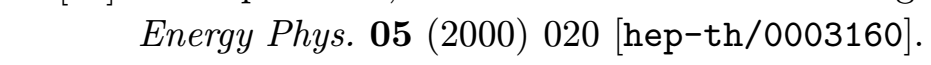

[27] H. Liu and J. Michelson, *-trek: the one loop $N=4$ noncommutative sym action, hep-th/0008205.

[28] D. Zanon, Noncommutative perturbation in superspace, hepeth/000

[29] F. Ardalan and N. Sadooghi, Anomaly and nonplanar diagrams in noncommutative gauge theories, hep-th/0009233.'

[30] M.R. Garousi, Non-commutative world-volume interactions on D-branes and DiracBorn-Infeld action, №cl. Phys. B 579 (2000) 209: thep-th/9909214].

[31] S. Hyun, Y. Kiem, S. Lee and C.-Y. Lee, Closed strings interacting with noncommutative D-branes, iNucl. Phys. B $\mathbf{5 6 9}(2000)-262$ [hep-th/9909059'].

[32] D.J. Gross, A. Hashimoto and N. Itzhaki, Observables of non-commutative gauge theories, hep-th/0008075:

[33] S.R. Das and S-J. Rey, Open Wilson lines in noncommutative gauge theory and tomography of holographic dual supergravity, 'Nucl. Phys. B- $\mathbf{5} \mathbf{9} \overline{0}-(2000)$ [hep-th/0008042in.

[34] N. Ishibashi, S. Iso, H. Kawai and Y. Kitazawa, Wilson loops in noncommutative Yang-Mills, iNucl. Phys. B 573 $(2000)$ 573, [hep-th/9910004.'.

[35] T. Asakawa and I. Kishimoto, Comments on gauge equivalence in noncommutative

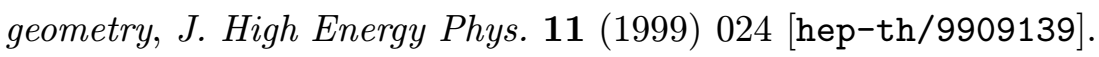

[36] K. Okuyama, A path integral representation of the map between commutative and noncommutative gauge fields, 'J. High Energy Phys. $\mathbf{0} 3$ (2000) 016' [hep-th/9910138].

[37] B. Jurco and P. Schupp, Noncommutative Yang-Mills from equivalence of star prod-

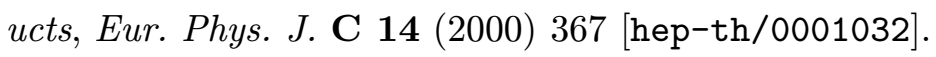

[38] B. Jurco, P. Schupp and J. Wess, Noncommutative gauge theory for poisson manifolds, iNucl. Phys. B 584 - 2000$)$ 784 [hep-th/0005005].

[39] B. Jurco, S. Schraml, P. Schupp and J. Wess, Enveloping algebra valued gauge trans-

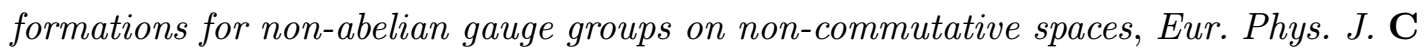

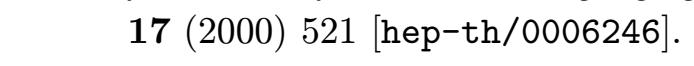

